
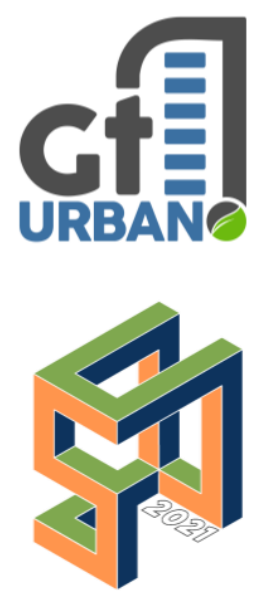

SINGEURB

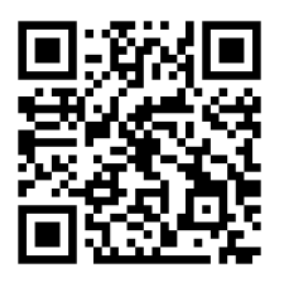

Como citar:

SENA, Filipe Batista; MARINS, Karin Regina de Castro. Análise alométrica das emissões de gases de efeito estufa nos municípios paulistas. In: III SIMPÓSIO NACIONAL DE GESTÃO E ENGENHARIA URBANA: SINGEURB, 2021, Maceió. Anais... Porto Alegre: ANTAC, 2021. p. 180186.

Disponível em: https://eventos.antac. org.br/index.php/sin geurb/issue/view/14

\title{
Análise alométrica das emissões de gases de efeito estufa nos municípios paulistas
}

\section{Allometric analysis of greenhouse gas emissions in brazilian municipalities}

Filipe Batista Sena, Universidade de São Paulo,

filipe.sena@usp.br

Karin Regina de Castro Marins, Universidade de São Paulo,

karin.marins@usp.br

\section{RESUMO}

Os inventários de emissões de gases de efeito estufa (GEE) são uma ferramenta importante no diagnóstico dos efeitos do meio urbano no meio ambiente e ponto de partida para proposição de estratégias de mitigação de mudanças climáticas. As metodologias para elaboração de inventários têm evoluído a partir da experiência, mas ainda apresentam divergências de resultados e dificuldades de serem inseridas no planejamento de políticas públicas. Assim, a busca por formas alternativas e mais acessíveis de analisar as emissões municipais de GEE é relevante, principalmente nas regiões onde a discussão ainda está em estágio preliminar. Este trabalho tem o objetivo de explorar uma abordagem de modelagem matemática baseada em alometria para previsão de emissões de GEE, no âmbito dos municípios do Estado de São Paulo. Os resultados aderem a resultados de aplicações similares de outros autores e apontam que quanto maior o município, maior a quantidade de emissões de GEE, proporcionalmente à população.

Palavras-chave: Mudanças climáticas, Alometria, Sistemas complexos.

\section{ABSTRACT}

Greenhouse gas (GHG) emission inventories are an important tool for diagnosing the effects of urban spaces on the environment and a starting point for proposing climate change mitigation strategies. The methodologies for elaborate inventories have evolved from experience, but they still present divergent results and difficulties in being included in public policies. Thus, the search for alternative and more accessible ways to analyze municipal GHG emissions is relevant, especially in regions where the discussion is still at a preliminary stage. This work aims to explore a mathematical modeling approach based on allometry to predict GHG emissions, in the scope of municipalities in the State of São Paulo, Brazil. The findings adhere to results from similar applications by other authors and point out that the larger the municipality, the greater the amount of GHG emissions, proportionally to the population.

Keywords: GHG Emissions, Allometry, Complex Systems. 


\section{INTRODUÇÃO}

As cidades já são reconhecidas como umas das principais fontes de emissão de gases de efeito estufa (GEE) (KENNEDY et al., 2010). A elaboração de Inventários municipais de GEE se tornou a principal forma de análise dos impactos urbanos e construção de planos para redução de consumo energético em longo prazo, assim como de outras atividades com emissões mais relevantes. As metodologias para Inventários de emissão GEE no nível municipal foram criadas com base nos inventários nacionais, mescladas com metodologias de inventários corporativos de empresas que analisam suas atividades. Esses ajustes metodológicos geram questões nas aplicações em escala municipal, pois as fronteiras e os objetos de análise são diferentes, quando se compara a dinâmica de uma cidade com a de uma empresa ou até mesmo de uma nação.

Em 2014, a ONG Local Governments for Sustainability (ICLEI) em parceria com o C40 lançaram o Global Protocol for Community-Scale Greenhouse Emission Inventories (GPC). O novo protocolo gerou expectativas e movimentou a discussão sobre inventários municipais, mas fica claro que este não apresenta novas soluções, apenas correções acerca das metodologias já existentes e demonstra que ainda não é consenso as melhores estratégias para elaborar inventários municipais (WILMSEN et al., 2016). As principais questões são as preferências por métodos top-down (bancos de dados) e bottom-up (levantamento em campo), escopos de emissões, correções de fatores de emissão utilizados. Diferentes protocolos divergem no cálculo de emissões e dificultam a comparação entre cidades (IBRAHIM et al., 2012).

Em regiões em que não existe uma cultura de levantamento permanente de dados, a falta de séries históricas e os custos iniciais para secretarias municipais são uma barreira para a obtenção de medições locais de emissões. Considerando que a mitigação de emissões urbanas de GEE seja o principal objetivo dos inventários, é necessário estabelecer estratégias que contornem as dificuldades existentes, dadas as metodologias propostas até o momento.

A alometria é o estudo da correlação entre as dimensões das partes corporais, como, por exemplo, tamanho do cérebro, vasos sanguíneos e porte do um ser vivo (WEST et al., 1997). Essa ciência tem origem na biologia, e atualmente tem uma analogia de metodologia como ferramenta de modelagem matemática para análise de sistemas complexos, como cidades, por exemplo. Bettencourt (2013) demostrou que diversas cidades americanas obedecem a relações alométricas em relação ao tamanho de sua população, densidade, área etc. Estudos discutem porque essas relações, chamadas funções livres de escala, aparecem em diversas cidades e em outros sistemas (NEWMAN, 2005). As relações de escala são identificadas por uma lei de potência da forma abaixo:

$Y=Y_{-} 0 X^{\wedge} \beta$, onde $Y_{-} 0$ e $\beta$ são constantes.

Segundo Bettencourt, para cidades os expoentes têm valores flutuando próximo de 1 . Quando o $\beta>1$, temos um "comportamento superlinear" e quando $\beta<1$ temos um "comportamento sublinear" ou chamado também de economia de escala (BETTENCOURT, 2007). Por exemplo, no trabalho de Bettencourt, a lei de potência que relaciona população da cidade com comprimento total de ruas tem $\beta=0,85$, ou seja, toda a vez que a cidade aumenta a população em $100 \%$, o comprimento total de ruas aumenta $85 \%$ de forma que cidades maiores economizam em infraestrutura em relação a cidades com menor população (BETTENCOURT, 2007). 
O objetivo da análise é identificar se existe uma expoente constante $(\beta)$ que relaciona os dados cruzados. Os gráficos na escala Log-Log, ou seja, ambos os eixos em escala logarítmica, apresentam tendência linear onde a inclinação da reta é o próprio expoente que rege a lei entre as duas variáveis (NEWMAN, 2005).

\section{METODOLOGIA}

Emissões de GEE relacionadas ao consumo de energia estacionária e transportes, equivalem a cerca de $80 \%$ das emissões totais das cidades (IBRAHIN et al., 2012; SUGAR et al., 2012). A energia estacionária é definida como o consumo elétrico de edifícios, residências e indústrias que geram emissões de GEE na fonte, enquanto o transporte se refere aos modos de transporte urbanos e são quantificados por consumo de combustíveis e fatores de emissão relativos a cada tipo de combustível. Utilizando o modelo de lei de potência, é possível analisar a relação entre emissões de GEE provenientes de consumo energético (estacionário e transporte) com diversos outros dados municipais, como a população. A comparação entre emissões e população, por exemplo, permite uma análise comparativa entre municípios de uma mesma região.

O anuário de emissões dos municípios paulistas de 2018, elaborado pela Secretaria de Energia e Mineração do Estado de São Paulo, apresenta dados das emissões referentes ao consumo energético e consumo de combustíveis dos 645 municípios do Estado (SIMA, 2021). As emissões de GEE foram comparadas com os dados de população, considerando as projeções para 2018 (SEADE, 2021), frota de veículos e PIB per capita fornecidos pelo IBGE, também para o ano de 2018, de forma a verificar a aderência dos dados ao modelo de lei de potência da equação (1).

O ano de 2018 foi considerado como referência devido à disponibilidade de dados e por ser anterior à pandemia de Covid-19, que pode distorcer aos dados devido aos eventos atípicos da série histórica.

\section{RESULTADOS}

No caso dos municípios paulistas, ambas as comparações de emissões de GEE com população e frota de veículos apresentaram comportamento superlinear. A comparação das emissões de GEE com o PIB municipal apresentou comportamento sublinear. A figura 1 apresenta os gráficos de correlação das emissões municipais de GEE com a população total (A), frota total (B) e Produto Interno Bruto (C). Sobre a figura também são apresentados os valores de $\beta$ de cada comparação e o coeficiente de determinação $\left(R^{2}\right)$ de cada regressão linear considerada. 
Figura 1 - Correlação entre Emissões de GEE, em toneladas de CO2eq e população total (A), frota total de veículos (B) e PIB (C), dos municípios do Estado de São Paulo
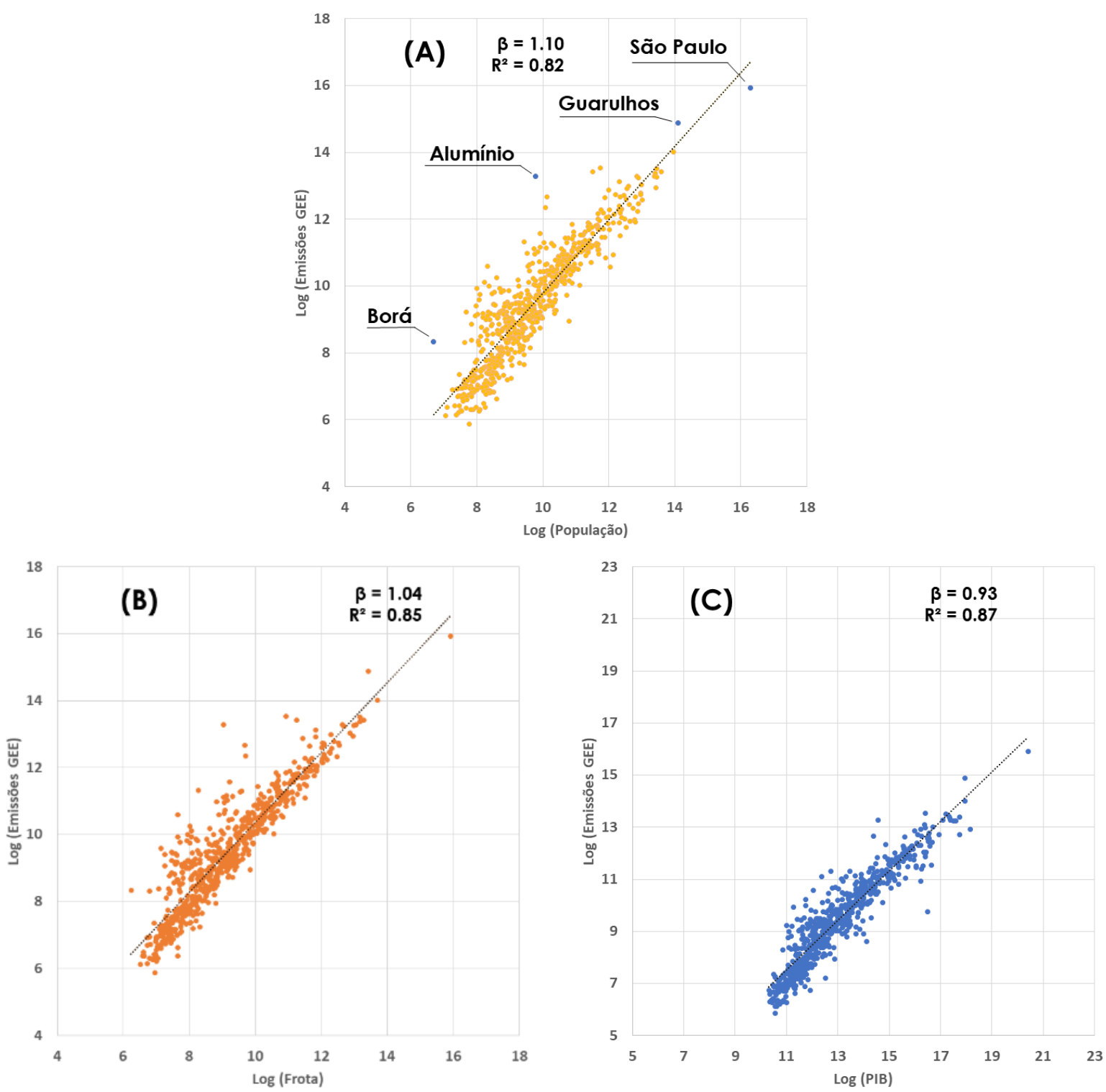

Fonte: Autores; SIMA, 2018; IBGE, 2018.

Ainda, desenvolver alternativas analíticas para o problema da mitigação de emissões de GEE em cidades é uma questão paralela a elaboração de inventários. Para regiões onde o levantamento de dados é limitado, a alometria pode ser usada como um ponto de partida para estratégia de combate às mudanças climáticas, onde a análise top-down compara cidade e calcula seu desvio ao modelo, para entender quais características urbanas e da população geram divergências para cima ou para baixo da previsão. A figura 2 e figura 3 apresentam um exemplo de como o modelo pode ser usado, indicando quais municípios estão acima ou abaixo da tendência (emissões de GEE são maiores ou menores que o previsto pelo modelo matemático). 
Figura 2 - Correlação entre Emissões de GEE e população, destacando os municípios que estão acima e abaixo da tendência

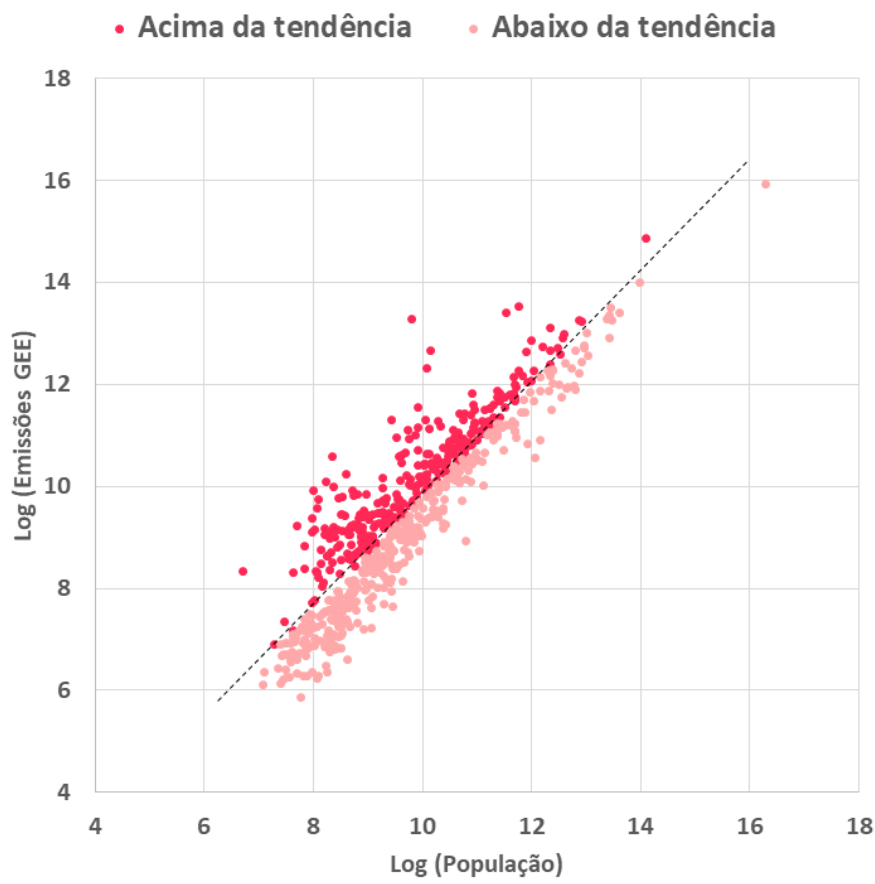

Fonte: Autores; SIMA, 2018; IBGE, 2018

Figura 3 - Municípios paulista em relação a tendência de emissão de GEE

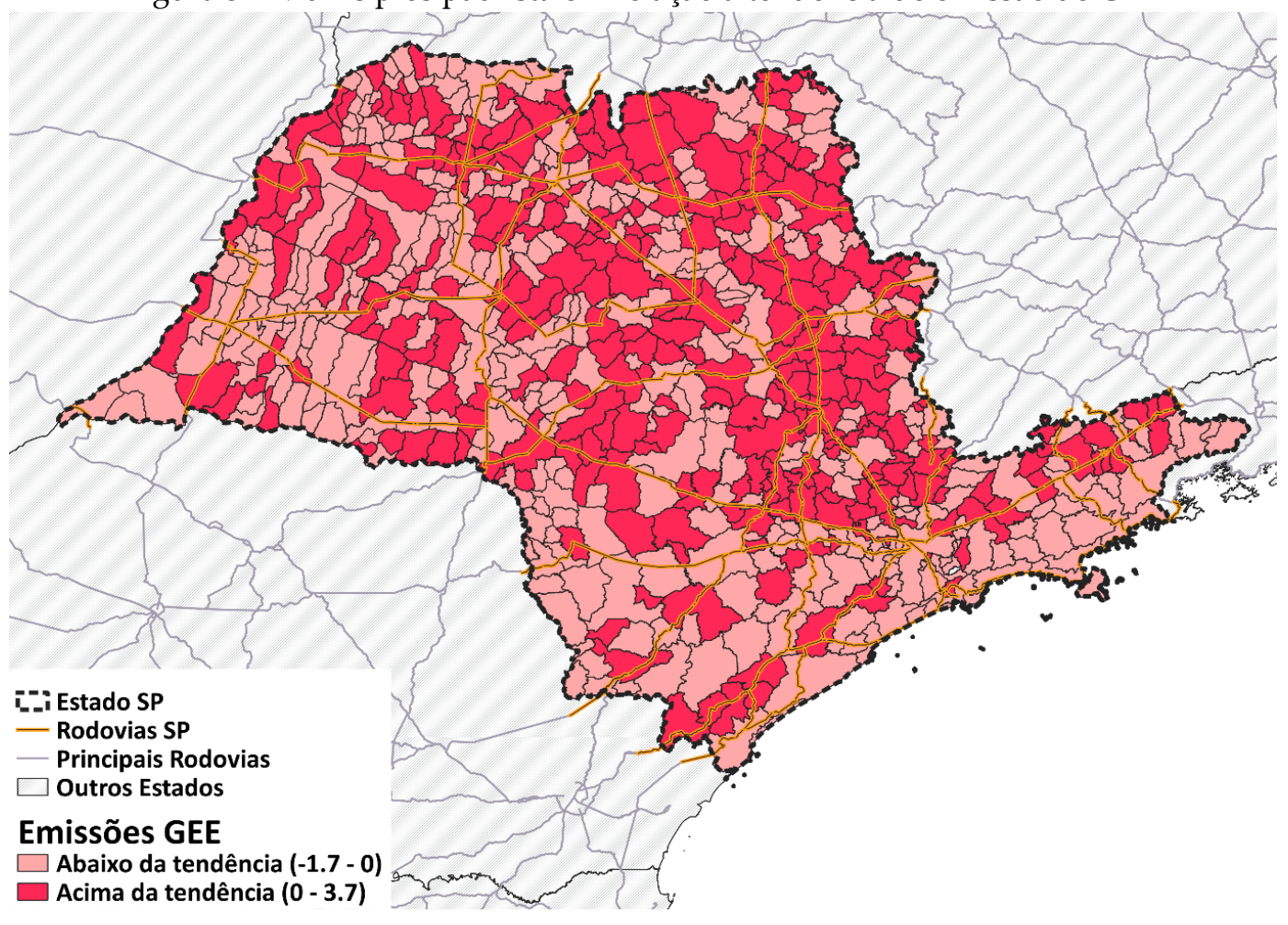

Fonte: Autores 


\section{CONCLUSÕES}

O resultado encontrado na análise de que cidades maiores emitem mais GEE, proporcionalmente a sua população, também aparece em outros estudos (SUGAR et al., 2012; OLIVEIRA e ANDRADE, 2014).

Entretanto, os valores dos expoentes encontrados nesses estudos divergem, ou seja, existe correlação com a população total dos municípios porém os valores de $\beta$ encontrados são diferentes, assim como a metodologia de análise e computação dos dados. O processo de desenvolvimento das cidades assim como suas atividades dependem de uma série de variáveis. Os estudos análogos de alometria feitos nos últimos anos, levam em consideração os efeitos emergentes das cidades que surgem das interações sociais, e apresentam características de sistemas complexos (BETTENCOURT et al., 2007). A diferença entre o valor reportado de emissões no município e o previsto na lei de escala pode ser expressão das ações dos agentes locais que variam em cada cidade. Estudar essas variações e entender como cada cidade pode contribuir para explicar o fenômeno de emissões de GEE, podem direcionar próximas etapas, em estudos dessa natureza. O PIB aborda outras atividades além do consumo de energia, como transações financeiras e comércio de produtos, sendo necessário uma investigação mais abrangente sobre este resultado.

Por meio da análise da linha de tendência e sua espacialização regional, podem ser definidas regiões prioritárias para levantamentos bottom-up, diminuindo-se custos individuais do amplo levantamento detalhado em âmbito municipal, sem saber seu real impacto na escala global. O modelo pode ser melhorado, por exemplo, por meio da análise matemática de outros indicadores, a fim de refinar as conclusões sobre emissões nos municípios paulistas.

\section{REFERÊNCIAS}

BETTENCOURT L. M. A.; LOBO J.; HELBING D.; KUHNERT C.; WEST G.B. Growth, innovation, scaling, and the pace of life in cities. Proc Natl Acad Sci. 2007;104(17):7301-6.

BETTENCOURT L. M. A. The Origins of Scaling in Cities. Science. 2013; Vol. 340. 1438-1444 (June).

INSTITUTO BRASILEIRO DE GEOGRAFIA E ESTATÍSTICA - IBGE. Frota de veículos 2006-2020. IBGE, 2021. Acesso em: 09 de junho de 2021. Disponível em: https://cidades.ibge.gov.br/brasil/pesquisa/22/28120?ano=2018.

INSTITUTO BRASILEIRO DE GEOGRAFIA E ESTATÍSTICA - IBGE. Produto Interno Bruto dos Municípios 2002-2018. IBGE, 2021. Acesso em: 09 de junho de 2021.Disponível em: https://www.ibge.gov.br/estatisticas/economicas/contas-nacionais/9088-produto-interno-bruto-dosmunicipios.html?=\&t=o-que-e

IBRAHIM N.; SUGAR L.; HOORNWEG D.; CHRISTOPHER K. Greenhouse Gas Emissions from cities: Comparison of International Inventory Frameworks. Local Environment. 2012; Vo. 17. 223-241.

KENNEDY C.; STEINBERGER J.; GASSON B.; HANSEN Y.; HILLMAN T.; HAVRANEK M. et al. Methodology for inventorying greenhouse gas emissions from global cities. Energy Policy. 2010;38(9):482837.

NEWMAN M. E. J. Power laws, Pareto distributions and Zipf's law. Contemp Phys. 2005;46(5):323-51. 
OLIVEIRA E. A.; ANDRADE J. S.; MAKSE H. A. Large cities are less green. Sci Rep. 2014; 4:13-21.

FUNDAÇÃO SISTEMA ESTADUAL DE ANÁLISE DE DADOS - SEADE. Projeções Populacionais para municípios paulistas 2012-2050. SEADE, 2021. Acesso em: 09 de junho de 2021. Disponível em: http://produtos.seade.gov.br/produtos/projpop/

SECRETARIA DE INFRAESTRUTURA E MEIO AMBIENTE - SIMA. Ranking Paulista de Energia 20122019. 2021. SIMA, 2021. Acesso em: 09 de junho de 2021. Disponível em: http://dadosenergeticos.energia.sp.gov.br/portalcev2/index.html

SUGAR L.; KENNEDY C.; LEMAN E. Greenhouse Gas Emissions from Chinese Cities. Jornal of Industrial Ecology. 2012;16(4):552-63.

WEST G. B.; BROWN J. H.; ENQUIST B.J. General Model for the Origin of Allometric Scaling Laws in Biology. Science. 1997;276(April):122-126.

WILMSEN F.; GESING F.; WILMSEN F.; GESING F. The Global Protocol for Community-Scale Greenhouse Gas Emission Inventories (GPC) - A New Passage Point on an Old Road? 2016;(68). 